\title{
Systems biology analysis of metabolism, signaling and gene expression regulation in human skeletal muscle
}

\author{
Ilya R. Akberdin \\ BIOSOFT.RU, LLC \\ FRCenter Institute of Cytology and \\ Genetics SB RAS \\ Novosibirsk State University \\ Novosibirsk, Russia \\ akberdinir@gmail.com \\ Alexander Yu. Vertyshev \\ CJSC "Sites-Tsentr" \\ Moscow, Russia \\ avertyshev@mail.ru
}

\author{
Ilya N. Kiselev \\ BIOSOFT.RU, LLC \\ Institute of Computational \\ Technologies SB RAS \\ Novosibirsk, Russia \\ axec@developmentontheedge.com \\ Pavel A. Makhnovskii \\ Institute of Biomedical Problems of the \\ RAS, Moscow, Russia \\ maxpauel@gmail.com
}

Fedor A. Kolpakov

BIOSOFT.RU, LLC

Institute of Computational

Technologies SB RAS

Novosibirsk, Russia

fkolpakov@gmail.com

\author{
Sergey S. Pintus \\ BIOSOFT.RU, LLC \\ Institute of Computational \\ Technologies SB RAS \\ Novosibirsk, Russia \\ sspintus@developmentontheedge.com \\ Daniil V. Popov \\ Institute of Biomedical Problems of the \\ RAS, Moscow, Russia \\ danil-popov@yandex.ru
}

\begin{abstract}
Physical exercise training induces profound molecular genetic adaptations in skeletal muscle cells. The adaptation is ensured by instant/transient activation of signaling pathways in the muscle cells eventually resulting in an alteration of both global metabolic network fluxes and expression of various genes. Despite the experimentally based efforts to disentangle the complexity of the muscle adaptation network caused by multiple interactions and intersections on signaling, metabolic and genetic regulatory levels, the quantitative and mechanistic contribution of each component of the signaling cascades on downstream genetic regulation processes has not been fully elucidated. Data-driven mathematical models provide a rigorous way to analyze and understand such intricate biological systems. Herein a novel integrated mathematical model linking intracellular metabolism, $\mathrm{Ca}^{2+}$-dependent signaling pathway and downstream transcription regulation of early and late response genes in human skeletal muscle during and after acute exercise developed in BioUML platform has been presented.
\end{abstract}

Keywords - mathematical model, skeletal muscle, physical exercise, $\mathrm{Ca}^{2+}$-dependent signaling pathway, $\mathrm{RNA}$ sequencing, regulation of expression, BioUML

\section{Introduction}

Skeletal muscle comprises about $40 \%$ of total body mass in adult lean humans and plays a crucial role in the control of whole-body metabolism and exercise tolerance. Regular lowintensity exercise (aerobic or endurance training) strongly induces adaptive changes in skeletal muscle which are increased vascular and mitochondrial density, oxidative capacity and improve fat and carbohydrate metabolism. These adaptations lead to an enhancement of muscle endurance performance and reduce the risk associated with the morbidity and premature mortality of chronic cardiovascular and metabolic diseases $[1 ; 2]$.

It is worth to note, although advancement in the development of high-throughput experimental techniques and generation of diverse omics data for human skeletal muscle during endurance exercise enabled to unveil key participants of the cellular response and adaptation [3-7], the system understanding of signaling-metabolic pathways relationships with downstream genetic regulation in exercising skeletal muscle is still elusive. As a complementary theoretical counterpart to the experimental investigation of molecular mechanisms underlying the skeletal muscle adaptation to the endurance training, a detailed mechanistic mathematical model provides a powerful in silico tool to quantitatively investigate signal transduction pathways and corresponding molecular mechanisms orchestrating gene expression dynamics during an exercise [8-11].

\section{Methwods}

\section{Model reconstruction}

We have previously developed a multi-compartmental mathematical model describing the dynamics of intracellular species concentrations and fluxes in human muscle at rest and intracellular metabolic rearrangements in exercising skeletal muscles during an aerobic exercise on a cycle ergometer [10]. As an initial model, we have used a complex model of energy metabolism in the human skeletal muscle developed by $\mathrm{Li}$ and coauthors [8]. We have proposed a modular representation of the complex model using BioUML platform [12]. To reconstruct the coupled signaling-gene expression regulation networks in SBGN standard [13] and build corresponding detailed mechanistic models we employed BioUML platform too [12]. The processes are distributed in two cellular compartments: signaling transduction pathway and translation, post-translation modification of the protein encoded by early response gene are represented in the cytosol, while the genetic regulation processes are located in the nucleus. The modular models of $\mathrm{Ca}^{2+}$-dependent signaling pathway and AMPK isoforms activities induced by the muscle construction are represented by ordinary differential equations (ODEs).

\section{Model validation and parameter fitting}

For our model, values of the almost all kinetic parameters were extracted from previous experimental data and mathematical models describing different aspects of the metabolic network, AMPK- and $\mathrm{Ca}^{2+}$-dependent signaling pathway [7-8; 14-19]. We adjusted the values of parameters that had not been reported in the published data to achieve consistency with experimental quantitative data on transcriptional activity PGC- $1 \alpha$ mRNA and a time-scale required to reach the maximal level of PGC- $1 \alpha$ mRNA in skeletal muscle after the endurance exercise as well as a time-scale to recover the basal level of transcriptional 
activity at the rest [7]. To simulate the muscle adaptation to endurance training we used experimentally verified shift of $\mathrm{Ca}^{2+}$ concentration which corresponds five-fold increase of the parameter value during the transition from rest to the exercise [11]. The numerical simulations of the dynamic model developed as a system of ordinary differential equations have been conducted using VODE method implemented in BioUML platform [12]. Sensitivity analysis of the model parameters and initial values of the model variables were performed using default settings in the corresponding BioUML module.

\section{RNAseq and proteomics data analysis}

Source of RNAseq data and procedure of data selection has been presented in our previous publication [7]. Original data on diverse content of proteins in slow and fast fibres were extracted from the Supplementary Material of the paper [14]. If a protein was not presented in this data set its concentration was calculated in the basis of RNAseq data due to the fact that protein concentration correlates with RPKM and FPKM values. FPKM values from the RNAseq data were used as a basis and compared to GTEx, Average RPKM values data for corresponding proteins in the skeletal muscles extracted from The Human Protein Atlas (www.proteinatlas.org). As a control of the calculation's correctness, a protein content in the skeletal muscle cell estimated using RNAseq data was compared to published proteomics data [14]. Results of calculations for such lowpresented proteins as subunits of AMPK, Calmodulin, CaMKII have demonstrated similar values of the concentrations estimated by two approaches. According to this for other low-presented proteins (i.e. transcription factors) concentrations estimated on the basis of RNAseq data were used if they were absent in proteomics study.

\section{Results}

The computational model describing a gene expression activation elicited by upstream signaling network during the transition from rest to exercise in human skeletal muscle has been developed based on modular modeling approach [12]. In turn, exercise-induced changes in gene expression levels promote the metabolic adaptation of the skeletal muscle cells by means of an activation mechanism which enhances energy metabolism via transport and reaction fluxes. In order to the integrated model represents actual changes in gene expression in exercised human skeletal muscle in more details, we replaced the general work rate parameter on the concentration of $\mathrm{Ca}^{2+}$-Calmodulin complexes and incorporated PGC- $1 \alpha-$ mediated transcription regulation of genes playing an important role in adaptation to regular exercise. In silico simulations demonstrate a high potential of the developed model to predict a proper cellular response to exercise based on the comparison between experimental measurements and simulated values of the model variables on all hierarchical levels (metabolic, signaling and gene expression regulation).

\section{ACKNOWLEDGMENT}

The study has been financially supported by RFBR grants (No. 17-00-00308 (K) and No. 17-00-00296).

\section{REFERENCES}

[1] B.K. Pedersen and M.A. Febbraio, "Muscles, exercise and obesity: skeletal muscle as a secretory organ," Nature Reviews Endocrinology, vol. 8, № 8, pp. 457-465, 2012.

[2] J.A. Hawley, M. Hargreaves, M.J. Joyne and J.R. Zierath, "Integrative biology of exercise," Cell, vol. 159, № 4, pp. 738-749, 2014.

[3] O. Neubauer et al., "Time course-dependent changes in the transcriptome of human skeletal muscle during recovery from endurance exercise: from inflammation to adaptive remodeling," Journal of Applied Physiology, vol. 116, № 3, pp. 274-287, 2013.

[4] K. Vissing and P. Schjerling, "Simplified data access on human skeletal muscle transcriptome responses to differentiated exercise," Scientific data, vol. 1, pp. 140041, 2014.

[5] D.V. Popov, P.A. Makhnovskii, N.S. Kurochkina, E.A. Lysenko, T.F. Vepkhvadze and O.L. Vinogradova, "Intensity-dependent gene expression after aerobic exercise in endurance-trained skeletal muscle," Biology of sport, vol. 35, № 3, pp. 277, 2018.

[6] J.M. Dickinson et al., "Transcriptome response of human skeletal muscle to divergent exercise stimuli.," Journal of Applied Physiology, vol. 124, № 6, pp. 1529-1540, 2018.

[7] D.V. Popov, et al., "Contractile activity-specific transcriptome response to acute endurance exercise and training in human skeletal muscle," American Journal of Physiology-Endocrinology and Metabolism, vol. 316, № 4, pp. E605-E614, 2019.

[8] Y. Li, R.K. Dash, J. Kim, G.M. Saidel, M.E. Cabrera, "Role of $\mathrm{NADH} / \mathrm{NAD}+$ transport activity and glycogen store on skeletal muscle energy metabolism during exercise: in silico studies," American Journal of Physiology-Cell Physiology, vol. 296, № 1, pp. 25-46, 2009.

[9] Akberdin I.R., Kazantsev F.V., Ermak T.V., Timonov V.S., Khlebodarova T.M., Likhoshvai V.A. In Silico Cell: Challenges and Perspectives// Mathematical Biology and Bioinformatics. 2013. V. 8. № 1. P. 295-315.

[10] Kiselev I.N., Akberdin I.R., Vertyshev A.Y., Popov D.V. and Kolpakov F.A. A modular visual model of energy metabolism in human skeletal muscle// Mathematical Biology and Bioinformatics. 2019. V. 14. № 2. P. 373-392.

[11] I.R. Akberdin, A.Y. Vertyshev, S.S. Pintus, D.V. Popov and F.A. Kolpakov. A mathematical model linking $\mathrm{Ca}^{2+}$-dependent signaling pathway and gene expression regulation in human skeletal muscle," Mathematical Biology and Bioinformatics, vol. 14, № 2, pp. 373-392, 2020.

[12] F.A. Kolpakov et al., "BioUML: an integrated environment for systems biology and collaborative analysis of biomedical data," Nucleic Acids Research, vol. 47, № W1, pp. W225-W233, 2019.

[13] N. Le Novere et al., "The systems biology graphical notation," Nature Biotechnology, vol. 27, № 8, pp. 735, 2009.

[14] M. Murgia et al., "Single muscle fiber proteomics reveals fiber-typespecific features of human muscle aging," Cell Reports, vol. 19, № 11, pp. 2396-2409, 2017.

[15] C.C. Carroll, J.A. Carrithers, T.A. Trappe, "Contractile protein concentrations in human single muscle fibers," Journal of Muscle Research and Cell Motility, vol. 25, № 1, pp. 55-59, 2004.

[16] M. Wilhelm et al., "Mass-spectrometry-based draft of the human proteome," Nature, vol. 509, № 7502, pp. 582, 2014.

[17] F. Edfors et al., "Gene-specific correlation of RNA and protein levels in human cells and tissues," Molecular systems biology, vol. 12, № $10,2016$.

[18] T.E. Jensen, J.F.P. Wojtaszewski and E.A. Richter, "AMP-activated protein kinase in contraction regulation of skeletal muscle metabolism: necessary and/or sufficient?," Acta physiologica, vol. 196, № 1, pp.155-174, 2009.

[19] R. Kjøbsted et al., "AMPK in skeletal muscle function and metabolism”, The FASEB journal, vol. 32, №4, pp.1741-1777, 2018. 